\title{
EVALUACIÓN DE LA EXPOSICIÓN OCUPACIONAL A CICLOFOSFAMIDA EN NUEVE HOSPITALES DEL PERÚ
}

\author{
Jaime A. Rosales-Rimache1,a
}

RESUMEN

\begin{abstract}
Objetivos. Evaluar la exposición ocupacional a ciclofosfamida en nueve hospitales del Perú. Materiales y métodos. Estudio observacional de tipo transversal realizado durante el año 2010 en el cual se colectó muestras de orina de $24 \mathrm{~h}$ de 96 trabajadores de las unidades de mezclas oncológicas y servicios de oncología de nueve hospitales del Perú, la cuantificación de ciclofosfamida se realizó por la metodología de GC-MS (Gas Cromathography-Mass Spectroscopy). Se realizó, además, muestreo de superficies de trabajo utilizando paños húmedos para la determinación de ciclofosfamida. Resultados. Se detectó la presencia de ciclofosfamida en orina en 67 trabajadores (concentración promedio de excreción: $74,2 \mathrm{ng} / 24 \mathrm{~h}$ ), lo cual representa el $70 \%$ del total de la población evaluada. Basado en la excreción se puede clasificar la exposición total entre los hospitales, en términos de exposición alta (>18,9 ng/24 h), moderada $(1,725-18,9 \mathrm{ng} / 24 \mathrm{~h})$ y baja $(<1,725 \mathrm{ng} / 24 \mathrm{~h})$, con una frecuencia porcentual de 31,$3 ; 26,0$ y $42,7 \%$ respectivamente. Además, como parte de la evaluación ambiental, se encontró concentraciones de ciclofosfamida de 14,72, 14,98 y 5,12 ng/cm². Conclusiones. Se evidencia una contaminación por ciclofosfamida en las áreas donde se realizan los preparados oncológicos y la presencia de ciclofosfamida en muestras de orina de trabajadores expuestos a citostáticos.
\end{abstract}

Palabras clave: Monitoreo del ambiente; Exposición ocupacional; Citostáticos (fuente: DeCS BIREME).

\section{EVALUATION OF OCCUPATIONAL EXPOSURE TO CYCLOPHOSPHAMIDE IN NINE HOSPITALS OF PERU}

\begin{abstract}
Objetives. Evaluate occupational exposure to cyclophosphamide in nine hospitals of Peru. Materials and methods. Crosscutting observational study conducted in 2010, for which 24-hour urine samples were obtained from 96 employees of the oncologic mixture units and oncology services of nine hospitals in Peru, the quantification of cyclophosphamide was done through the GC-MS methodology (Gas Cromathography-Mass Spectroscopy). Additionally, working surfaces were tested by obtaining samples with wet wipes for identification of cyclophosphamide. Results. Cyclophosphamide was detected in urine samples in 67 employees (average concentration of excretion: $74.2 \mathrm{ng} / 24 \mathrm{~h}$ ), accounting for $70 \%$ of the total population to be assessed. Based on the excretion, total exposure among hospitals can be classified as high level (>18.9 ng/24 h), moderate level $(1,725-18.9 \mathrm{ng} / 24 \mathrm{~h})$ and low level $(<1,725 \mathrm{ng} / 24 \mathrm{~h})$, with a percent incidence of $31.3 ; 26.0$ and $42.7 \%$ respectively. Additionally, as part of the environmental evaluation, concentrations of cyclosphamide were found in $14.72,14.98$ and 5,12 $\mathrm{ng} / \mathrm{cm} 2$. Conclusions. Contamination through cyclophosphamide in areas where oncological preparations are done and the presence of cyclophosphamide in urine samples of workers exposed to cytostatics substance were observed.
\end{abstract}

Key words: Environmental monitoring; Occupational exposure; Cytostatic agents (source: MeSH NLM).

\section{INTRODUCCIÓN}

En el Perú, el principal tratamiento de cáncer es la quimioterapia basada en la administración de antineoplásicos o fármacos citostáticos cuyo uso es cada vez más cotidiano, lo que conlleva a que el personal que manipula estas sustancias químicas aumenten su riesgo de exposición. Se sabe bien que la gran mayoría de citostáticos interactúan con el ácido desoxirribonucleico o sus precursores inhibiendo la síntesis del nuevo material genético ${ }^{(1)}$.

Para el uso de estos fármacos se realiza una previa formulación, preparación y administración que es llevada a cabo por trabajadores de salud, quienes están en riesgo de exposición a estos medicamentos, el cual se incrementa de no cumplirse los criterios de bioseguridad para el manejo de estos compuestos ${ }^{(2-4)}$. El problema

Centro Nacional de Salud Ocupacional y Protección del Ambiente para la Salud, Instituto Nacional de Salud. Lima, Perú.

Licenciado tecnólogo médico, magíster en Salud Ocupacional

Recibido: 10-07-2013 Aprobado: 02-10-13

Citar como: Rosales-Rimache JA. Evaluación de la exposición ocupacional a ciclofosfamida en nueve hospitales del Perú. Rev Peru Med Exp Salud Publica. 2013;30(4):590-4. 
que se presenta por la exposición ocupacional es que pueden ocasionar efectos mutagénicos, carcinogénicos y teratogénicos ${ }^{(5,6)}$.

El biomonitoreo ocupacional se constituye como la mejor herramienta para evaluar la exposición biológica a citostáticos, es también importante el monitoreo en ambientes de trabajo ${ }^{(7-9)}$. La concentración urinaria del citostático o de sus metabolitos se determina usando un método de análisis químico. Debido a la reactividad química, a las vías de biotransformación complejas y al esperado bajo nivel de exposición, los niveles de citostáticos o de sus metabolitos en orina que pueden encontrarse son bajos, por lo que son necesarios métodos muy sensibles como la espectroscopia de masas ${ }^{(10)}$. De acuerdo con muchas publicaciones internacionales se indica que el método más específico y sensible para evaluar la exposición a citostáticos es la determinación de ciclofosfamida en orina (8-14); considerando que es el fármaco que tiene mayor uso dentro de los esquemas de tratamiento antineoplásico.

El objetivo de la presente investigación fue evaluar la exposición ocupacional a ciclofosfamida, en cinco hospitales y establecer la exposición a ciclofosfamida en trabajadores de salud expuestos a citostáticos de nueve hospitales del Perú.

\section{MATERIALES Y MÉTODOS}

\section{DISEÑO Y POBLACIÓN DE ESTUDIO}

Estudio observacional de tipo transversal. La recolección de datos fue realizada durante el año 2011 siguiendo un muestreo no probabilístico, la población estuvo constituida por 96 trabajadores de salud que realizaban la preparación y formulación de las mezclas oncológicas y otras que se dedicaban a la administración de la quimioterapia en los servicios de oncología. La población procedía de nueve hospitales, siendo cinco hospitales de Lima, tres hospitales de regiones de la zona sur y un hospital de la zona norte del Perú.

\section{TOMA DE MUESTRAS BIOLÓGICAS}

Se colectó 96 muestras de orina de trabajadores de los nueve hospitales. Las muestras de orina fueron colectadas durante un periodo de $24 \mathrm{~h}$ usando los kits Cyto Urine. Todas las muestras fueron almacenadas bajo congelamiento $\left(-20^{\circ} \mathrm{C}\right)$ después de la colección y durante el transporte hasta la preparación de las muestras. El análisis de ciclofosfamida (seis mediciones por trabajador evaluado) fue realizado por el Laboratorio Exposure Control B.V. en Holanda.

\section{TOMA DE MUESTRAS AMBIENTALES}

Se realizó utilizando paños húmedos embebidos en una solución de $\mathrm{NaOH} 0,03 \mathrm{M}$, después de haber concluido la jornada laboral, los cuales fueron tomados en 30 puntos distintos de cinco hospitales que otorgaron el permiso institucional para realizar el muestreo ambiental (hospital 1, 2, 3, 4 y 6), donde se calculó el tamaño de las posiciones y de las áreas muestreadas (medición del ancho y largo del área muestreada expresada en centímetros). Los paños fueron tomados con los kits Cyto Wipe proveídos por el Laboratorio Exposure Control B.V. de Holanda. Los puntos de muestreo fueron seleccionados considerando su ubicación dentro de las áreas donde se realiza la preparación de las mezclas oncológicas (en algunos hospitales, con la presencia de cabinas de bioseguridad y en otros donde no se cuenta con barreras de contención). El citostático analizado fue la ciclofosfamida expresada en cantidad del mismo por área muestreada $\left(\mathrm{ng} / \mathrm{cm}^{2}\right)$.

\section{TÉCNICAS ANALÍTICAS}

El análisis de ciclofosfamida se realizó usando la metodología de cromatografía de gasesespectroscopia de masas (GC-MS) ${ }^{(10)}$. Sin embargo, las muestras que resultaron con valores bajos (cercanos a $0,10 \mathrm{ng} / \mathrm{mL} \mathrm{NaOH}$ ) fueron analizadas con un sistema de cromatografía de gases acoplada a espectroscopía de masas en tándem (GC-MSMS) usada para separar compuestos orgánicos de una mezcla o solución ${ }^{(13)}$. La sensibilidad y especificidad se incrementa por el uso del sistema GC-MSMS en comparación al GC-MS.

\section{ANÁLISIS ESTADÍSTICO}

El análisis estadístico se realizó a través del uso del software SPSS v 19.0 en la cual se hizo el cálculo de los estadísticos descriptivos.

\section{CONSIDERACIONES ÉTICAS}

Todos los participantes fueron informados de los procedimientos a realizar, riesgos, beneficios y la confidencialidad de los resultados. Los participantes otorgaron su consentimiento informado voluntariamente. Posteriormente, se procedió a la entrega de los resultados toxicológicos en las personas evaluadas, a quienes se les explicó detalladamente sobre los mismos. 
Tabla 1. Características de los trabajadores de unidades de mezclas oncológicas y servicios de oncología de nueve hospitales del Perú, 2010

\begin{tabular}{lrrccc}
\hline Hospital & $\mathbf{N}$ & $\begin{array}{c}\text { Mujeres } \\
(\%)\end{array}$ & $\begin{array}{c}\text { Años prome- } \\
\text { dio de exposi- } \\
\text { ción laboral }\end{array}$ & $\begin{array}{c}\text { Unidad de } \\
\text { mezclas } \\
\text { oncológi- } \\
\text { cas }\end{array}$ & $\begin{array}{c}\text { Onco- } \\
\text { logía }\end{array}$ \\
\hline Hospital 1 & 11 & $11(100)$ & 3,0 & 2 & 9 \\
\hline Hospital 2 & 4 & $4(100)$ & 24,3 & 0 & 4 \\
\hline Hospital 3 & 15 & $15(100)$ & 7,0 & 2 & 13 \\
\hline Hospital 4 & 10 & $8(53)$ & 1,6 & 1 & 9 \\
\hline Hospital 5 & 11 & $9(82)$ & 4,5 & 11 & 0 \\
\hline Hospital 6 & 30 & $24(80)$ & 6,8 & 19 & 11 \\
\hline Hospital 7 & 1 & $0(0)$ & 2,0 & 1 & 0 \\
\hline Hospital 8 & 13 & $9(69)$ & 8,9 & 6 & 7 \\
\hline Hospital 9 & 1 & $0(0)$ & 7,0 & 1 & 0 \\
\hline
\end{tabular}

\section{RESULTADOS}

\section{CARACTERÍSTICAS DEMOGRÁFICAS}

El promedio del tiempo de exposición laboral en las personas evaluadas fue de 6,6 años, con un rango de 1 a 30 años, el 83,3\% (80/96) de los participantes eran mujeres, el 44,8\% (43/96) trabajaban en unidades de mezclas oncológicas y 55,2\% (53/96) en el servicio de oncología (Tabla 1).

\section{MONITOREO BIOLÓGICO}

Las cantidades totales de ciclofosfamida excretada por hospital fue desde 1,5 hasta 4419,5 ng/24 h. La cantidad promedio de ciclofosfamida excretada por trabajador fue de $74,2 \mathrm{ng} / 24 \mathrm{~h}$. de algunos trabajadores de salud, todas las muestras fueron positivas indicando más o menos una exposición continua a la ciclofosfamida. La cantidad promedio de ciclofosfamida excretada por trabajador por hospital va desde 11,6 hasta 1163,9 ng/24 h.

El número más elevado de muestras de orina positivas para ciclofosfamida se observó en los trabajadores del hospital $2(100 \%)$ y el hospital $5(91 \%)$. Se encontró un resultado similar en el hospital $9(100 \%)$, pero se refirió a la evaluación de un solo trabajador. No se detectó ciclofosfamida en las muestras de orina del único trabajador de la unidad de mezclas oncológicas del hospital 7 (Tabla 2). Los resultados de orina muestran exposición a ciclofosfamida en el $70 \%$ de los trabajadores evaluados.

\section{MONITOREO AMBIENTAL}

La contaminación con ciclofosfamida monitoreada en cinco hospitales encontró niveles elevados de
Tabla 2. Niveles de ciclofosfamida en muestras de orina de 96 trabajadores de unidades de mezclas oncológicas y servicios de oncología de nueve hospitales de Perú, 2010

\begin{tabular}{lrrrr}
\hline Hospital & $\mathbf{N}$ & $\begin{array}{c}\text { Trabajadores } \\
\text { con ciclofosfa- } \\
\text { mida urinaria } \\
\text { positiva (\%) }\end{array}$ & $\begin{array}{c}\text { Ciclofosfa- } \\
\text { mida total } \\
\text { por hospital } \\
\text { (ng/24 h) }\end{array}$ & $\begin{array}{c}\text { Ciclofosfamida } \\
\text { promedio por } \\
\text { trabajador por } \\
\text { hospital } \\
\text { (ng/24 h) }\end{array}$ \\
\hline Hospital 1 & 11 & $9(82)$ & 178,8 & 16,3 \\
Hospital 2 & 4 & $4(100)$ & 4655,6 & 1163,9 \\
\hline Hospital 3 & 15 & $7(47)$ & 918,6 & 61,2 \\
\hline Hospital 4 & 10 & $5(50)$ & 115,9 & 11,6 \\
\hline Hospital 5 & 11 & $10(91)$ & 534,2 & 48,6 \\
\hline Hospital 6 & 30 & $22(73)$ & 443,4 & 14,8 \\
\hline Hospital 7 & 1 & $0(0)$ & 0 & 0 \\
\hline Hospital 8 & 13 & $10(77)$ & 250,0 & 19,2 \\
\hline Hospital 9 & 1 & $1(100)$ & 26,0 & 26,0 \\
\hline
\end{tabular}

contaminación (1-10 $\left.\mathrm{ng} / \mathrm{cm}^{2}\right)$ en casi todas las cabinas de cuatro hospitales. Sobre otras posiciones, se midieron niveles bajos de contaminación. No se halló contaminación en las dos superficies de oncología de hospital 3 y una superficie en oficina de hospital 4 (Tabla 3).

Tabla 3. Evaluación ambiental de ciclofosfamida en unidades de mezclas oncológicas de cinco hospitales del Perú, 2010

\begin{tabular}{|c|c|}
\hline Descripción de la superficie & $\begin{array}{c}\text { Ciclofosfamida } \\
\left(\mathrm{ng} / \mathrm{cm}^{2}\right)\end{array}$ \\
\hline \multicolumn{2}{|l|}{ Hospital 1} \\
\hline Superficie interna de cabina (base) & 5,06 \\
\hline Superficie interna de cabina (superior) & 5,31 \\
\hline Superficie interna de cabina (ventana) & 0,74 \\
\hline \multicolumn{2}{|l|}{ Hospital 2} \\
\hline Pseudo cabina (vitrina interior) & 0,86 \\
\hline Pseudo cabina (vitrina exterior) & 0,04 \\
\hline \multicolumn{2}{|l|}{ Hospital 3} \\
\hline Superficie de mesa oncología & ND \\
\hline Superficie de pared oncología & ND \\
\hline Cabina 1 (interior 1 ) & 1,12 \\
\hline Cabina 1 (interior 2) & 0,19 \\
\hline Cabina 2 (interior 1 ) & 0,02 \\
\hline Cabina 2 (interior 2) & 4,32 \\
\hline \multicolumn{2}{|l|}{ Hospital 4} \\
\hline Cabina (vidrio interior) & 0,69 \\
\hline Cabina (interior) & 1,15 \\
\hline Pared (interior 1) & 0,04 \\
\hline Pared (interior 2) & 0,08 \\
\hline Pared (exterior) & 0,01 \\
\hline Pared (interior - oficina) & ND \\
\hline \multicolumn{2}{|l|}{ Hospital 6} \\
\hline Cabina 1 (interior) & 14,72 \\
\hline Cabina 2 (interior) & 14,98 \\
\hline Cabina 3 (interior) & 5,12 \\
\hline Puerta (interior) & 1,10 \\
\hline
\end{tabular}

ND: no detectado 


\section{DISCUSIÓN}

Los resultados muestran una contaminación generalizada con ciclofosfamida. La contaminación con ciclofosfamida tambien se observó en la unidad de mezclas oncológicas del hospital 1, en la unidad de mezclas oncológicas de la farmacia del hospital 3 y en menor extensión en el área de oncología del hospital 2 y en la unidad de mezclas oncológicas del hospital 4, sin embargo, los niveles de contaminación son más bajos comparados al presentado en la unidad de mezclas oncológicas del hospital 6.

Otros estudios también han mostrado contaminación ambiental con fármacos citostáticos en farmacias hospitalarias y áreas de administración del medicamento ${ }^{(8-10)}$. Los niveles más altos de contaminación fueron hallados dentro de las cabinas de bioseguridad. La contaminación de estas superficies indica derrames durante la preparación de estos fármacos. El derrame ocurre principalmente durante la preparación cuando el fármaco es transferido desde los viales a las jeringas y desde las jeringas hacia los sistemas intravenosos (sistemas de venoclisis). La diferencia de presión dificulta su manejo. El derrame y la consecuente contaminación deberían prevenirse tanto como sea posible para evitar la exposición a los trabajadores que se evaluaron en este estudio.

Basado en la excreción se puede clasificar la exposición total entre los hospitales, en términos de exposición alta (>18,9 ng/24 h); moderada (1,725 - 18,9 ng/24 h) y baja $(<1,725 \mathrm{ng} / 24 \mathrm{~h})$, con una frecuencia porcentual de 31,$3 ; 26,0$ y $42,7 \%$ respectivamente. Sin embargo, se debe considerar que en algunos casos solo se evaluó un trabajador con cantidades superiores de ciclofosfamida al del promedio excretado por el total de trabajadores que corresponden a un hospital.

Si las cantidades de ciclofosfamida que se han medido en este estudio se hubiesen excretado diariamente por un periodo prolongado (40 años de exposición), sería posible estimar el riesgo de cáncer por exposición a ciclofosfamida. Esto ha sido posible tomando como referencia la base de estudios epidemiológicos en modelos animales y pacientes que tuvieron tumores primarios y secundarios tratados con ciclofosfamida; datos que fueron extrapolados con la excreción de ciclofosfamida de 476 muestras de orina en trabajadores de farmacia con un rango de 3,6 a $18 \mu \mathrm{g} / \mathrm{día}$. Se determinó que el riesgo umbral para cáncer es de 1 en 1 millón, y el riesgo prohibitorio no debería exceder el valor de 100 por millón (11-12). Sin embargo, debería señalarse que se han hecho muchos supuestos hasta ahora.

Basados en la excreción promedio (por trabajador por hospital) de ciclofosfamida se encontró 11,6 a 1163,9 ng/día para este estudio, el riesgo de cáncer para los trabajadores del hospital 2, 3, 5 y 9 (un solo trabajador) está por encima del riesgo promedio que debería tolerarse ${ }^{(13)}$. El riesgo de cáncer para los trabajadores del hospital 1, 4 y 8 , está por debajo del nivel de riesgo tolerable. Está claro que en general la exposición a ciclofosfamida y otros fármacos citostáticos debería evitarse tanto como sea posible y lo ideal sería que no se detectará la presencia de estos citostáticos en las muestras de orina.

No se puede establecer con claridad las rutas de exposición para los trabajadores; sin embargo, los resultados de orina proporcionan algo de información. La exposición a ciclofosfamida por inhalación de partículas suspendidas en aire resulta en la forma más rápida de ingreso al organismo y, del mismo modo, en la excreción urinaria del citostático ${ }^{(14-18)}$. Por otro lado, la excreción a largo plazo de ciclofosfamida (muestras de orina positivas) corresponde a un ingreso del fármaco posterior y a la excreción de ciclofosfamida.

Es importante señalar que siendo este el primer estudio en el Perú que permite evidenciar y evaluar las condiciones laborales de personas ocupacionalmente expuestas a citostáticos a través de una evaluación biológica y ambiental, es recomendable que debe implementarse con urgencia un programa de vigilancia sanitaria para la evaluación de trabajadores expuestos a fármacos citostáticos; así como mejorar las condiciones de bioseguridad en las áreas que impliquen su manipulación y, sobre todo, definir un marco legal específico que regule el trabajo con estos fármacos, considerando que algunos de ellos son de naturaleza carcinogénica y no se encuentran incluidos en el listado oficial de sustancias carcinogénicas del Perú, problema que requiere la atención y el compromiso de las autoridades a nivel técnico, administrativo y político con una visión transectorial del estado.

En conclusión, los resultados en este estudio indican un problema muy serio de exposición ocupacional a ciclofosfamida en los hospitales evaluados. Los procedimientos de manejo de fármacos citostáticos deberían ser reevaluados y mejorados, además de contar con instructivos de limpieza adecuados y efectivos del área de trabajo para remover la contaminación remanente y evitar la exposición al trabajador.

Agradecimientos: al licenciado estadístico Marco Gonzáles Noriega por su apoyo en el análisis estadístico; a la técnica de laboratorio Jesús del Milagro Abadíe Timaná por el apoyo en la toma de muestras biológicas; así como al licenciado Carlos Mori Gupioc por el apoyo en la recolección de datos.

Conflictos de interés: el autor declara no tener conflictos de interés.

Fuentes de financiamiento: este estudio fue financiado por del Instituto Nacional de Salud. 


\section{REFERENCIAS BIBLIOGRÁFICAS}

1. Ursini CL, Cavallo D, Colombi A, Giglio M, Marinaccio A, Iavicoli S. Evaluation of early DNA damage in healthcare workers handling antineoplastic drugs. Int Arch Occup Environ Health. 2006 Nov;80(2):134-40.

2. España, Ministerio de Trabajo y Asuntos Sociales, Instituto Nacional de Seguridad e Higiene en el Trabajo. NTP 163: Exposición laboral a compuestos citostáticos [Internet]. Madrid: Ministerio de Trabajo y Asuntos Sociales; 1986 [citado 16 de octubre de 2012]. Disponible en: http:// www.insht.es/InshtWeb/Contenidos/ Documentacion/FichasTecnicas/ NTP/Ficheros/101a200/ntp_163.pdf

3. España, Ministerio de Trabajo y Asuntos Sociales, Instituto Nacional de Seguridad e Higiene en el Trabajo. NTP 740: Exposición Laboral a Citostáticos en el Ámbito Sanitario [Internet]. Madrid: Ministerio de Trabajo y Asuntos Sociales; 2006. [citado 16 de octubre de 2012]. Disponible en: http://www. insht.es/InshtWeb/Contenidos/ Documentacion/FichasTecnicas/ NTP/Ficheros/701a750/ntp_740.pdf

4. National Institute of Occupational Safety and Health (NIOSH); Center for Disease Control and Prevention (CDC). Preventing occupational exposures to antineoplastic and other hazardous drugs in health care settings. Cincinnati [Internet]. OH: NIOSH 2004 [citado el 16 de octubre de 2012]. Disponible en: http://www.cdc.gov/ niosh/docs/2004-165/pdfs/2004-165. pdf

5. World Health Organization, International Agency for Research on Cancer. IARC monographs on the evaluation of carcinogenic risks to humans. Overall evaluations of carcinogenicity: an Updating of IARC [Internet]. Geneva: WHO; 1987 [citado el 22 de octubre de 2012]. Disponible en: http://monographs. iarc.fr/ENG/Monographs/vol26/ volume26.pdf
6. World Health Organization, International Agency for Research on Cancer. IARC monographs on the evaluation of carcinogenic risks to humans. Overall evaluations of carcinogenicity: an Updating of IARC [Internet]. Geneva: WHO; 1997 [citado el 22 de octubre de 2012]. Disponible en: http://monographs. iarc.fr/ENG/Monographs/vol50/ index.php

7. Consejo Interterritorial del Sistema Nacional de Salud. Agentes Citostáticos. Protocolo de Vigilancia Sanitaria Específica [Internet]. Madrid: Ministerio de Sanidad y Consumo; 2003 [citado el 25 de octubre de 2012]. Disponible en: http://www.msssi.gob. es/ciudadanos/salud AmbLaboral/ docs/Agentescitostaticos.pdf

8. Sessink PJM, Scholtes MM, Anzion RBM, Bos RP. Determination of cyclophosphamide in urine by gas chromatography-mass spectrometry. J Chromatogr. 1993;616(2):333-7.

9. Sessink PJM, Anzion RBM, van den Broek PHH, Bos RP. Detection of contamination with antineoplastic agents in a hospital pharmacy department. Pharm Weekbl. 1992;14(1):16-22.

10. Sessink PJM,BoerKA,ScheefhalsAPH, Anzion RBM, Bos RP. Occupational exposure to antineoplastic agents at several departments in a hospital. Environmental contamination and excretion of cyclophosphamide and ifosfamide in urine of exposed workers. Int Arch Occup Environ Health. 1992;64(2):105-12.

11. Sessink PJM. Monitoring of occupational exposure to antineoplastic agents. Exposure Control; 1996.

12. Connor TH, Anderson RW, Sessink PJM, Broadfield L, Power LA. Surface contamination with antineoplastic agents in six cancer treatment centers in Canada and the United States. Am J Health-Syst Pharm. 1999; 56(14):1427-32.
13. Sessink PJM, Kroese ED, van Kranen HJ, Bos RP. Cancer risk assessment for health care workers occupationally exposed to cyclophosphamide. Int Arch Occup and Environ Health. 1995;67(5):317-23.

14. Hirst M, Tse S, Mills DG, Levin L. Occupational exposure to cyclophosphamide. Lancet. 1984;1(8370):186-8.

15. Sessink PJM, van de Kerkhof MCA, Anzion RBM, Noordhoek J, Bos RP. Environmental contamination and assessment of exposure to antineoplastic agents by determination of cyclophosphamide in urine of exposed pharmacy technicians: Is skin absorption an important exposure route? Arch Environ Health. 1994;49(3):165-9.

16. Evelo CTA, Bos RP, Peters JGP, Henderson PTh. Urinary cyclophosphamide assay as a method for biological monitoring of occupational exposure to cyclophosphamide. Int Arch Occup Environ Health. 1986;58(2):151-5.

17. Sessink PJM, Cerná M, Rössner P, Pastorková A, Bavarová $H$, Franková $\mathrm{K}$, Anzion RBM, Bos RP. Urinary cyclophosphamide excretion and chromosomal aberrations in peripheral blood lymphocytes after occupational exposure to antineoplastic agents. Mutat Res. 1994;309(2):193-9.

18. Ensslin AS, Stoll Y, Pethran A, Pfaller A, Römmelt H, Fruhmann G. Biological monitoring of cyclophosphamide and iphosfamide in urine of hospital personnel occupationally exposed to cytostatic drugs. Occup Environ Med. 1994;51(4):229-33.

Correspondencia: Jaime Rosales Rimache Dirección: Calle Las Amapolas 350, Lima 14 Teléfono: 7480077 anexo 7706, 7717 y 7725 Correo electrónico:jrosales@ins,gob,pe 